Check for updates

Cite this: RSC Adv., 2018, 8, 4063

\title{
Highly-sensitive detection of eight typical fluoroquinolone antibiotics by capillary electrophoresis-mass spectroscopy coupled with immunoaffinity extraction $\uparrow$
}

\begin{abstract}
Xiao-Hui Zhang, Yan Deng, Ming-Zhe Zhao, Ying-Lin Zhou (D) * and Xin-Xiang Zhang*
Fluoroquinolones (FQs) are a class of synthetic antibiotics with a broad active spectrum towards mycoplasma and bacteria (both Gram positive and negative). The contamination of environmental water samples with FQs has been a concern due to the extensive use of them in the veterinary and pharmaceutical industries. Reliable and simple analytical methods are needed for the monitoring of these FQs. An off-line procedure of immunoaffinity extraction (IAE) followed by capillary electrophoresis-mass spectroscopy (CE-MS) has been developed for simultaneous determination of eight typical FQs in environment water. A broad-specific polyclonal antibody was home-prepared and immobilized on a Sepharose 4B stationary phase for sample affinity extraction and the enrichment factor of our columns was about 100. CE-MS was subsequently applied to FQs separation and quantification. The immunoaffinity column was evaluated in the parameters of selectivity, binding capacity, elution protocol, and recovery. Under the optimized conditions, the developed method was successfully applied to analyze FQs spiked with $2.5 \mathrm{nM}$ to $30 \mathrm{nM}$ of environmental water from a local lake, providing satisfactory recoveries from $72 \%$ to $112 \%$, and LODs and LOQs ranging from $1.2 \mathrm{nM}$ to $5.0 \mathrm{nM}$ and $4.0 \mathrm{nM}$ to $17 \mathrm{nM}$, respectively.
\end{abstract}

Received 18th November 2017
Accepted 14th January 2018

DOI: $10.1039 / \mathrm{c} 7 \mathrm{ra12557g}$

rsc.li/rsc-advances chromatography with fluorescence detection (HPLC-FD), ${ }^{5-8}$ immunoassay, ${ }^{\mathbf{9}, 10}$ HPLC with ultraviolet (UV) ${ }^{\mathbf{1 1}}$ or mass spectroscopy (MS) ${ }^{12,13}$ detection, capillary electrophoresis (CE) both with $\mathrm{UV}^{\mathbf{1 4 , 1 5}}$ or $\mathrm{FD}{ }^{16}$ and flow injection method with chemiluminescence detection. ${ }^{17}$ In our previous work, ${ }^{18}$ we detected five typical FQs in low-fat milk by field-enhanced sample injection-based CE in bubble cell capillary. As an online sample stacking strategy to overcome the low loading capacity of CE, field-enhanced sample injection was performed at the boundary of sample matrix with low-conductivity and the background electrolyte (BGE) zone with high-conductivity. That's to say such a developed method is not suitable to detect FQs in high-salt contained matrix like environmental water. Among those, immunoassay and methods using optical detectors did not provide structure confirmatory analysis, while the instrumentbased methods combining separation techniques and MS identification require an effective sample cleanup procedure to remove the complex matrix interference and extract the analytes.

The reported cleanup methods for FQs include solid phase extraction (SPE), ${ }^{\mathbf{6}, 11,12,15}$ pressurized liquid extraction (PLE), ${ }^{\mathbf{1 9 , 2 0}}$ matrix solid-phase dispersion (MSPD), ${ }^{21,22}$ microwave-assisted extraction, ${ }^{23,24}$ microdialysis. ${ }^{25}$ The most common used SPE was reverse-phase SPE based upon the hydrophobic interaction between the analytes and the sorbents, which was lack of
Beijing National Laboratory for Molecular Sciences (BNLMS), MOE Key Laboratory of Bioorganic Chemistry and Molecular Engineering, College of Chemistry, Peking University, Beijing 100871, China. E-mail: zhouyl@pku.edu.cn; zxx@pku.edu.cn; Fax: +86-10-62754112; Tel: +86-10-62754112

$\dagger$ Electronic supplementary information (ESI) available. See DOI: $10.1039 / \mathrm{c} 7 \mathrm{ra12557g}$ 
selectivity, leading to the co-extraction of analytes and matrix interference, especially when interested analytes are at trace levels and interference at higher concentration. A special SPE, named immunoaffinity extraction (IAE) based on the highly specific recognition between antigen and antibody, allows for a high degree of molecular selectivity. ${ }^{26}$ IAE was generally realized by loading a large volume of the sample into the immunoaffinity column and afterwards washing the unbounded or nonspecifically adsorbed interferences, eluting the specifically captured compounds for the subsequent qualitative or quantitative analysis. This strategy has been applied in environmental monitoring, ${ }^{27,28}$ pharmaceutical and biomedical analysis, ${ }^{29-31}$ and food analysis. ${ }^{32,33}$ As a specific extraction technique, IAE has been successfully used to online enrich four FQs in milk $^{34}$ prior to HPLC-FD, providing a satisfactory enrichment with good selectivity.

In this work, a generic antibody for a group of immunological similar FQs was prepared for immunoaffinity column formation to simultaneously extract those FQs analogs selectively. By combining IAE and CE-MS, detecting 8 FQs simultaneously was achieved. Samples were specifically purified and concentrated by IAE. The eluent from immunoaffinity column was concentrated and subsequently analyzed by CE-MS. We applied this method to determine FQs spiked water sample from a local lake. The LODs ranged from $1.2 \mathrm{nM}$ to $5.0 \mathrm{nM}$, which matched the result of the National standard method, HPLC-FD, with the advantages at simplicity and structural specificity.

\section{Experimental section}

\subsection{Reagents and solutions}

Bovine serum albumin (BSA), ovalbumin (OVA), Tween-20, incomplete Freund's adjuvant, and complete Freund's adjuvant were purchased from Sigma (St. Louis, MO, USA). $\mathrm{N}$-Hydroxysuccinimide (NHS) and 1-(3-dimethylaminopropyl)-3ethyl carbodiimide (EDC) were from Alfa Aesar (Johnson Matthey, USA). 3,3',5,5'-Tetramethylbenzidine (TMB), and horseradish peroxidase labeled goat anti-rabbit IgG (HRP-SecAb) were obtained from Balb Corp. (Beijing, China). Defatted milk was purchased from the local supermarket. Ciprofloxacin (CIP) and enrofloxacin (ENR) were from TCI (Tokyo, Japan), and pefloxacin (PEF), ofloxacin (OFL), gatifloxacin (GAT), fleroxacin (FLE), lomefloxacin (LOM) and enoxacin (ENO) from National Institutes for Food and Drug Control (Beijing, China). Methanol was of HPLC grade and obtained from Avantor (Avantor Performance Materials, Inc. USA). All other chemicals, including formic acid (FA), acetic acid (AA), ethylenediamine (EDA), hydrogen peroxide $\left(\mathrm{H}_{2} \mathrm{O}_{2}\right)$, quinine (QUIN), and ethylenediamine (EDA) were of analytical grade or better and obtained from Beijing Chemical Reagent Co. (Beijing, China). Unless specified, all buffers were prepared in high purity water from a Milli-Q purification system (Millipore, Bedford, MA, USA).

In enzyme-linked immunosorbent assay (ELISA), $0.01 \mathrm{M}$ phosphate buffered saline (PBS, $\mathrm{pH}$ 7.4) was prepared. $0.05 \mathrm{M}$ $\mathrm{Na}_{2} \mathrm{CO}_{3}-\mathrm{NaHCO}_{3}$ (CBS, pH 9.6) was used as coating buffer. Blocking solution was made by dissolving $5 \mathrm{~g}$ of milk in $100 \mathrm{~mL}$ of PBS, and washing buffer (PBST) consisted of $250 \mu \mathrm{L}$ of Tween20 and $500 \mathrm{~mL}$ of PBS. The substrate solution was prepared before use by mixing $0.1 \mathrm{M}$ phosphate buffer (PB, pH 6.0), TMB (6 mg mL $\mathrm{m}^{-1}$ in DMSO) and $\mathrm{H}_{2} \mathrm{O}_{2}(30 \%)$ at a volume ratio of $1000: 20: 2$.

Stock solutions of the above mentioned FQs and QUIN were prepared in $20 \mathrm{mM} \mathrm{NaOH}$ or $1 \mathrm{mM} \mathrm{HCl}$ at a concentration of $10 \mathrm{mM}$ and stored at $-20{ }^{\circ} \mathrm{C}$. The working solutions were prepared by diluting the stock solutions with assay buffer.

\subsection{Apparatus}

CE was performed using a PA 800 plus System (Beckman Coulter Inc., USA) equipped with a UV detector. MS analyses were realized by a 6320 Ion Trap (Agilent Technologies Inc., USA) with a commercial ESI source. The coupling of CE to MS was carried out using a G1608A Agilent CE-MS sprayer kit (Agilent Technologies Inc., USA), which is arranged orthogonally to MS inlet capillary and constituted of a triple-tube design accommodating one tube for separation capillary, one for sheath liquid delivery and another one to provide the nebulizing gas. All UV absorbance was measured on a HITACHI U4100 spectrophotometer (Tokyo, Japan), and all polystyrene 96-well microtiter plates (Costar, USA) were read on Multiskan FC Microplate Photometer (Thermo Fisher Scientific, Waltham, MA, USA).

\subsection{Preparation and characterization of broad-specific antibody against FQ analogs}

2.3.1 Synthesis of immunogen and coating antigen. CIP was selected because of its common molecular structure. It was coupled with BSA (CIP-BSA) to immunize the rabbits, with OVA (CIP-OVA) using as coating antigen in ELISA. The conjugation procedure was based on the previously reported NHS ester method $^{35}$ with slightly modifications. In particular, CIP (90 mg) was dissolved in DMF (30 mL), followed by the addition of EDC $(120 \mathrm{mg})$ and NHS $(120 \mathrm{mg})$ to obtain the active NHS solution. The mixture was stirred at room temperature overnight. The carrier proteins (140 mg BSA, and $90 \mathrm{mg}$ OVA) were dissolved in $30 \mathrm{~mL}$ carbonate buffer $(0.1 \mathrm{M}, \mathrm{pH} 8.0)$, respectively. The above active NHS mixture was added dropwise to the carrier protein solutions with continuous stirring and further stirred at room temperature for $4 \mathrm{~h}$. After being dialyzed against PBS for $12 \mathrm{~h}$ and deionized water for $24 \mathrm{~h}$ at $4{ }^{\circ} \mathrm{C}$ to remove the unbound hapten and residual solvent, the conjugate was lyophilized to white powder and stored at $-20{ }^{\circ} \mathrm{C}$ before use. The ratio of CIP and protein in immunogen was estimated through the molecular weights of BSA and CIP-BSA determined by MALDITOF-MS.

2.3.2 Production of polyclonal antibody. New Zealand rabbits were purchased from Xinglong experimental animal farm (Beijing). Three healthy New Zealand rabbits were immunized with the immunogen, CIP-BSA. The immunization procedure involved five subcutaneous injections at different body parts on rabbits. ${ }^{36}$ Anti-serum was obtained seven days after the last injection and purified based on a modified method of octanoic acid-saturated ammonium sulfate. ${ }^{37}$ All the 
experiments conform to the US National Institutes of Health "Guide for Care and Use of Laboratory animals" and were approved by the Institutional Animal Care and Use Committee of Peking University. The concentration of IgG was $16 \mathrm{mg} \mathrm{mL}{ }^{-1}$, which was estimated by its absorbance at $280 \mathrm{~nm}$. The purified $\mathrm{pAb}$ was stored at $-20{ }^{\circ} \mathrm{C}$.

2.3.3 Titer level and affinity constant of the purified antibody. The optimization of coating concentration of CIP-OVA and the determination of titer level of purified pAb were performed by ELISA based on a chessboard method. ${ }^{37}$ First of all, a 96-well microplate was coated with $100 \mu \mathrm{L}$ of coating buffer containing CIP-OVA at different concentrations overnight at $4{ }^{\circ} \mathrm{C}$. Afterwards, the microplate was blocked with $200 \mu \mathrm{L}$ blocking solution ( $5 \%$ milk) at $37^{\circ} \mathrm{C}$ for $2 \mathrm{~h}$. Next, $100 \mu \mathrm{L}$ of tenfold serially diluted purified pAbs were added to each well and incubated for $1 \mathrm{~h}$ at $37^{\circ} \mathrm{C}$. The followed is the addition of $100 \mu \mathrm{L}$ HRP-SecAb (1: 5000 diluted) for $1 \mathrm{~h}$ at $37^{\circ} \mathrm{C}$, then with $100 \mu \mathrm{L}$ substrate solution added to react for $15 \mathrm{~min}$ in dark. The enzyme reaction was stopped by $50 \mu \mathrm{L}$ of $2 \mathrm{M} \mathrm{H}_{2} \mathrm{SO}_{4}$ before the absorbance of each well read at $450 \mathrm{~nm}$.

The affinity constant was estimated in the process of optimizing coating concentration using the equation presented by Beatty. $^{38}$

2.3.4 Cross-reactivity of polyclonal antibody with CIP analogs. The specificity of the obtained anti-CIP pAb was investigated by testing its cross-reactivities with the other seven FQs and QUIN by competitive ELISA. The microplate was coated with $100 \mu \mathrm{L}$ of CIP-OVA at an optimized concentration overnight at $4{ }^{\circ} \mathrm{C}$, and blocked by defatted milk as described above. $50 \mu \mathrm{L}$ competitive compounds were then added at various concentrations together with $50 \mu \mathrm{L}$ of the $10^{4}$-fold diluted purified pAb. Other steps were the same as described in Section 2.3.3.

\subsection{Preparation of immunoaffinity column}

The immunoaffinity column was prepared according to the method reported before. ${ }^{29} 0.3 \mathrm{~g}$ CNBr-activated Sepharose 4B was swelled and washed with $1 \mathrm{mM} \mathrm{HCl}$, and then dispersed in $5 \mathrm{~mL}$ coupling buffer (0.1 $\mathrm{M} \mathrm{NaHCO}_{3}, 0.5 \mathrm{M} \mathrm{NaCl}, \mathrm{pH} 8.3$ ) containing $18 \mathrm{mg}$ pAb. The coupling of pAb to Sepharose 4B was performed in a rotated vessel for $2.5 \mathrm{~h}$ at room temperature. After the slurry was transferred into an injector $(5 \mathrm{~cm} \times 0.7 \mathrm{~cm}$ id), the excess antibody was removed from the column by eluting with the coupling buffer. The unreacted groups in the gel were blocked with $0.1 \mathrm{M}$ Tris- $\mathrm{HCl}$ buffer $(\mathrm{pH}$ 8.0) at room temperature for $2 \mathrm{~h}$. The column was then washed for three times by repeating the cycles of $5 \mathrm{~mL}$ of $0.1 \mathrm{M} \mathrm{NaAc}-\mathrm{HAc}$ buffer (containing $0.5 \mathrm{M} \mathrm{NaCl}$, pH 4.0) followed by $5 \mathrm{~mL}$ of $0.1 \mathrm{M}$ Tris$\mathrm{HCl}$ buffer (containing $0.5 \mathrm{M} \mathrm{NaCl}, \mathrm{pH}$ 8.0). $0.01 \mathrm{M}$ PBS was finally applied for the column equilibration and preservation.

\subsection{Procedure of immunoaffinity solid-phase extraction}

$10 \mathrm{~mL}$ sample was loaded on the immunoaffinity column, which has already been conditioned with $0.01 \mathrm{M}$ PBS. The column was then washed with $3 \mathrm{~mL}$ PBS to remove the nonspecifically captured analytes and subsequently washed by $3 \mathrm{~mL}$ water to reduce the negative effect of salt on CE-MS. After eluting the immunoaffinity column with $5 \mathrm{~mL}$ methanol, the collected effluent was concentrated to about $1 \mathrm{~mL}$ by high purity nitrogen and transferred to centrifugal vacuum concentrator (Eppendorf, Germany) for further concentration and drying. The extracted analyte was re-dissolved in deionized water $(100 \mu \mathrm{L})$ before the following CE-MS analysis.

\subsection{CZE separation coupled with ESI-MS detection}

Fused-silica capillaries (50/375 $\mu \mathrm{m}$ id/od, 40/50 $\mathrm{cm}$ effective/ total length) (Ruifeng Inc., Hebei, China) were used for FQs separation. Capillary zone electrophoresis conditions were set as follows: applied voltage, $25 \mathrm{kV}$; sample injection, 0.5 psi for $5 \mathrm{~s}$; capillary temperature, $25{ }^{\circ} \mathrm{C}$. All data were collected at $280 \mathrm{~nm}$. Before the first use, the capillaries were conditioned by sequentially rinsing by 20 psi with $1 \mathrm{M} \mathrm{NaOH}$ for $30 \mathrm{~min}$, deionized water for $15 \mathrm{~min}$ and running buffer for $5 \mathrm{~min}$. Between consecutive analyses, the capillary was only rinsed with running buffer for $4 \mathrm{~min}$. The running buffer was $50 \mathrm{mM}$ EDAFA ( $\mathrm{pH}$ 8.0).

When MS was hyphenated with CE via a coaxial sheath liquid interface for post-column detection, the length of the fusedsilica capillary was extended to $65 \mathrm{~cm}$. The sheath liquid was composed of methanol/water $(50: 50 \mathrm{v} / \mathrm{v})$ containing $0.1 \%(\mathrm{v})$ FA, and introduced at a flow rate of $5 \mu \mathrm{L} \mathrm{min}{ }^{-1}$ by a syringe pump. An electrospray potential of $-4.0 \mathrm{kV}$ was applied at the inlet of the MS (positive mode) while the CE-MS interface was grounded. A nebulizing gas pressure of 5.0 psi was provided to assist the spraying. Drying gas temperature and flow were set at $270{ }^{\circ} \mathrm{C}$ and $2.0 \mathrm{~L} \mathrm{~min}^{-1}$, respectively.

Unless specified, all solutions for CE-MS were filtered through $0.22 \mu \mathrm{m}$ membrane and degassed by ultrasonication before use.

\subsection{Real sample analysis}

Fresh water was collected from Weiming Lake at Peking University (Beijing, China), and used to dilute the standard FQs solution to defined concentrations. FQs spiked samples were immediately filtered through $0.22 \mu \mathrm{m}$ cellulose nitrate membrane filters (Jinteng, China) before extraction in order to prevent the influence of the matrix from fresh water on immunoaffinity column. The blank water sample and the spiked real samples (both in $10 \mathrm{~mL}$ ) were performed in the same way by the developed IAC and CE-MS off-line coupling procedure.

\section{Results and discussion}

\subsection{Synthesis of artificial antigen}

Antigen designing plays the most crucial role in antibody preparation, especially in the case of producing a broad-specific antibody. Herein, our aim lies in preparing an antibody that could immunologically recognize eight FQs and developing an immunoaffinity solid-phase extraction system for simultaneous enrichment of these FQs. Thus, the hapten, which was proposed to conjugate with a carrier protein to generate an immunogen for antibody selection, should retain the most of common 
structural features in FQ analogs. One or more FQs has been reported to be used as a hapten to produce a generic antibody, ${ }^{1}$ and CIP might be the desirable hapten considering its availability and reliability. The immunogen, CIP-BSA, was prepared through CIP conjugated to BSA with the NHS method and the coating antigen was prepared through CIP coupled with OVA in the same way. The conjugation ratio of CIP and BSA was estimated to be 11.7 via the molecule weights of BSA $(66.4 \mathrm{kDa})$ and its conjugation with CIP (70.3 kDa).

\subsection{Antibody characterization}

The purified polyclonal antibody was assessed by ELISA in the respect of titer level, affinity constant and cross-reaction.

The initial concentrations $\left(C_{0}\right)$ of the antibody was $16 \mathrm{mg} \mathrm{mL}^{-1}$ by UV measurement. The optimal concentration for coating was estimated by coating the microtiter plates with $100 \mu \mathrm{L}$ CIP-OVA of different concentrations $\left(2 \mu \mathrm{g} \mathrm{mL} L^{-1}, 4 \mu \mathrm{g} \mathrm{mL}{ }^{-1}, 5 \mu \mathrm{g} \mathrm{mL} L^{-1}\right.$, $6 \mu \mathrm{g} \mathrm{mL} \mathrm{m}^{-1}, 8 \mu \mathrm{g} \mathrm{mL} \mathrm{L}^{-1}$ and $\left.10 \mu \mathrm{g} \mathrm{mL}{ }^{-1}\right)$. According to the shape of sigmoid curves in Fig. 1(a), $8 \mu \mathrm{g} \mathrm{mL} \mathrm{m}^{-1}$ was chosen as optimal concentration for CIP-OVA coating, and the titer of this purified pAb was $1: 10^{4.8}$ (pAb dilution corresponding to $50 \%$ of the maximum absorbance).

Affinity constant $\left(K_{\text {aff }}\right)$ of antibody represents the combination stability of immunocomplex. Herein, $K_{\text {aff }}$ of pAb with
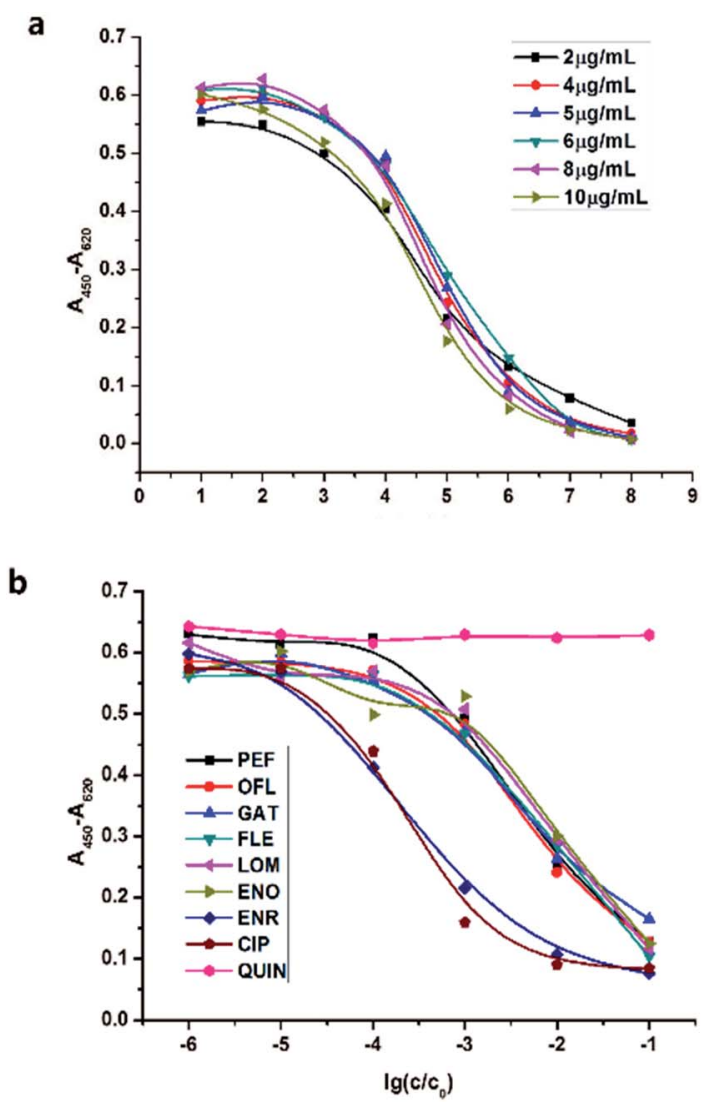

Fig. 1 (a) Coating concentration optimization of CIP-OVA and titer level determination of antibody by ELISA. (b) Cross-reactivities of pAb with eight FQ analogs by competitive ELISA (QUIN as control test).
CIP-OVA can be calculated as $1.53 \times 10^{7} \mathrm{~L} \mathrm{~mol}^{-1}$ in the optimization procedure of the coating concentration, which was high enough for immunoaffinity enrichment.

The broad-specificity of antibody was determined by a screening competitive ELISA (Fig. 1(b)) and calculated according to $50 \%$ displacement method. ${ }^{39}$ The microtiter plates were coated with $100 \mu \mathrm{L}$ CIP-OVA $\left(8 \mu \mathrm{g} \mathrm{mL} \mathrm{mL}^{-1}\right)$. The purified pAb was diluted by $10^{4}$-fold before addition $(50 \mu \mathrm{L}$ per microwell). The initial concentrations $\left(C_{0}\right)$ of the analytes (including QUIN for control test) were $0.1 \mathrm{mM}$. The crossreactivity of this purified pAb with CIP was defined as $100 \%$, and it was found to be $10 \%$ with PEF, $10 \%$ with OFL, $10 \%$ with GAT, $10 \%$ with FLE, $8 \%$ with LOM, 10\% with NOR, $8 \%$ with ENO, and $100 \%$ with ENR, indicating its broad-specificity to all these eight FQs.

\subsection{Evaluation of the immunoaffinity column}

The total amount of pAb immobilized in $0.3 \mathrm{~g}$ of $\mathrm{CNBr}-$ Sepharose $4 \mathrm{~B}$ was estimated to be $17.7 \mathrm{mg}\left(59 \mathrm{mg} \mathrm{g}^{-1}\right)$ by measuring the concentrations of pAb before and after the coupling reaction. The final gel volume of the affinity column (dead volume) was about $2.0 \mathrm{~mL}$. In order to investigate retention behavior of CIP in the immunoaffinity column, breakthrough curve of CIP $\left(0.4 \mu \mathrm{g} \mathrm{mL}^{-1}\right.$ in PBS $)$ was compared with that of QUIN (0.4 $\mu \mathrm{g} \mathrm{mL}^{-1}$ in PBS). As shown in Fig. 2(a), QUIN was detected in the first effluent fraction after discarding the dead volume of $2.5 \mathrm{~mL}$. In contrast, CIP was strongly retained in the column, and the breakthrough of CIP was not observed until $5.5 \mathrm{~mL}$, which demonstrated the specific retention of CIP in the immunoaffinity column. Binding capacity, another critical parameter in immunoaffinity column, is defined as the maximal amount of antigen captured by immobilized antibodies, and overloading the capacity would lower the accuracy of quantitative analysis. The theoretical binding capacity was calculated to be $20 \mu \mathrm{g}$ according to the amount of immobilized antibody and its two antigen binding sites without consideration of the antibody inactivation. Otherwise, the experimental value of binding capacity was obtained as $1.03 \mu \mathrm{g}$ by overloading the column with CIP $(20 \mu \mathrm{g}$, $1 \mathrm{~mL}$ ) and measuring the uncaptured (washed by PBS) or captured (eluted by methanol) target. It was much lower than the theoretical one, mainly resulted from the steric hindrance between analyte and antibody, as well as the random orientation and inactivation of the antibody.

\subsection{Elute conditions}

The antigen-antibody complex, formed via electrostatic forces, hydrogen bonds and van der Waals forces, was generally disrupted by low-pH solution or water-organic modifier mixture to dissociate the analytes without adversely affecting the immunological activity of antibodies. Here, high percentage of an organic solvent was chosen to dissociate captured small molecules efficiently considering the following two aspects. First, a large volume of low-pH solution was required for complete dissociation $^{29}$ which was not suitable for off-line procedure. In addition, the volatility of organic solvent would 


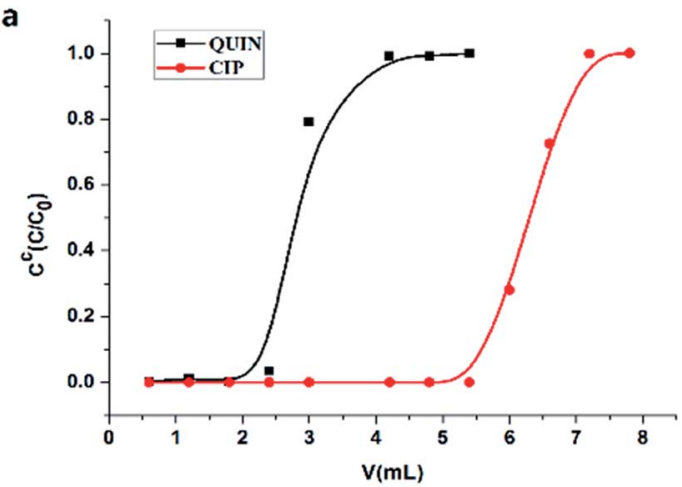

b

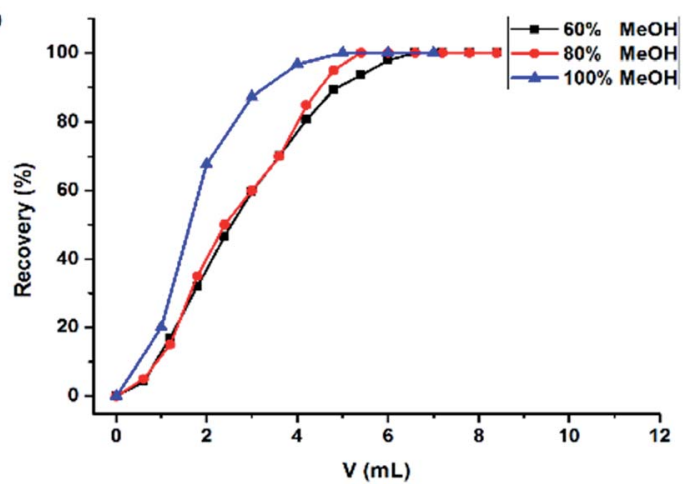

Fig. 2 (a) Breakthrough curves of CIP and QUIN obtained in anti-CIP pAb immobilized column. Sample concentration was $0.4 \mu \mathrm{g} \mathrm{mL}^{-1}$ both for CIP and QUIN. (b) Elution profiles of CIP with different percent of methanol.

make the off-line pre-concentration easier for the subsequent CE-MS analysis. The actual methanol concentration needed relied on the affinity constant between the analytes and the immobilized antibody. Thus, different percentages of methanol in water $(60 \%, 80 \%, 100 \%)$ were investigated to make a compromise between complete elution of the analytes and minimal damage to the antibody. After the immuno-capture of CIP $\left(20 \mu \mathrm{g} \mathrm{mL} \mathrm{m}^{-1}\right)$, the elution fraction was collected in one Eppendorf tube after another and detected by UV respectively. The recovery of target compound increased with the solvent volume for eluting (Fig. 2(b)). All captured compound was completely eluted under three conditions, demonstrating a good target release performance. Nevertheless, the total volume of water-methanol mixture required for thorough dissociation decreased along with the increasing of methanol percentage. Moreover, the immobilized antibody was not observed to be damaged even when $100 \%$ methanol was applied for elution owing to the short exposing time of the antibody to organic solvent. Decrease of enrichment efficiency of our columns was not observed after about 50 times use. For the process efficiency and simplicity, $100 \%$ methanol was chosen as the optimum elution solvent. The elution volume was increased to be $5 \mathrm{~mL}$ rather than $4 \mathrm{~mL}$ to ensure a complete dissociation of the immuno-complexes of analytes and immobilized antibody.

\subsection{CZE separation with UV or MS detection for quantitative analysis}

Basic BGE is generally reported for $F Q s$ separation. ${ }^{15,40}$ Considering its compatibility with ESI-MS, the BGE should be constituted of volatile salts such as ammonium carbonate. ${ }^{41}$ However, FQs cannot be separated completely by using ammonium carbonate. Recently, BGE based on EDA with pH 8.0 adjusted by $\mathrm{FA}^{18}$ was proposed as volatile and compatible with MS system for FQs separation. FQs were detected in positive mode in CE-MS and quantitative ions for MS detection were listed in Table S1. $\dagger$ As indicated in Fig. 3(a), the considered eight FQs can be basely separated under optimized conditions within 15 min both for UV and MS detection. Theoretically, CEMS was more time-consuming than CE-UV due to its longer separation channel and lowered electric field intensity, which cannot be found in the electropherograms. This can be explained by the syphonic effect between the two ends of CE separation capillary when there was no outlet vial assembled in CE equipped with MS detector.

The mixtures of eight FQs at different concentrations were prepared and analyzed both by CE-UV and CE-MS to investigate the linearity of the proposed procedure. The dependence of FQs peak heights (obtained in CE-UV) and peak areas (obtained in CE-MS) on their concentrations were fitted linearly, respectively,
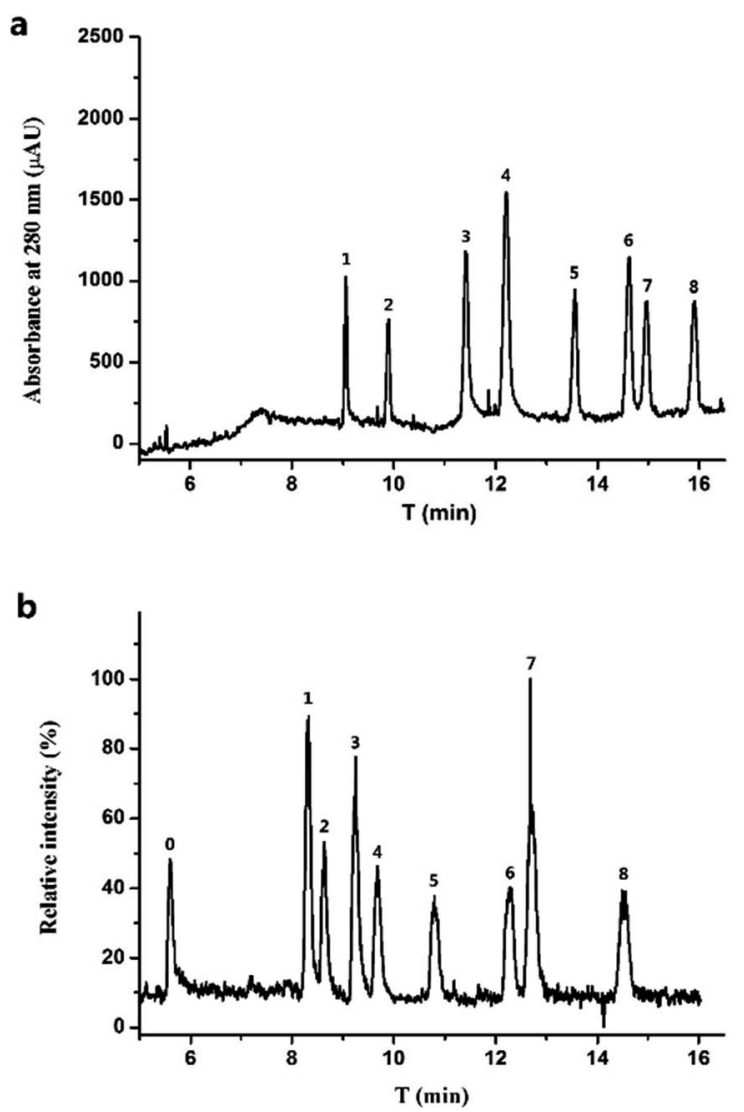

Fig. 3 Electropherograms with UV detection (a) and MS detection (b) of eight FQs (20 $\mu \mathrm{M}$ for each) and QUIN $(5 \mu \mathrm{M})$. Peaks are labelled as follows: QUIN as 0; GAT as 1; LOM as 2; ENO as 3; CIP as 4; OFL as 5; ENR as 6; FLE as 7; PEF as 8. 
Table 1 Calibration curves and LODs of FQs obtained in CE-MS under the optimized conditions

\begin{tabular}{|c|c|c|c|c|c|c|}
\hline \multirow[b]{2}{*}{ FQs } & \multicolumn{3}{|l|}{ CE-MS } & \multicolumn{3}{|l|}{ CE-UV } \\
\hline & $\begin{array}{l}\text { Calibration } \\
\text { curves }\end{array}$ & $\begin{array}{l}\text { Calibration } \\
\text { range }(\mu \mathrm{M})\end{array}$ & $\begin{array}{l}\text { LOD/LOQ } \\
(\mu \mathrm{M})\end{array}$ & Calibration curves & $\begin{array}{l}\text { Calibration } \\
\text { range }(\mu \mathrm{M})\end{array}$ & $\begin{array}{l}\text { LOD/LOQ } \\
(\mu \mathrm{M})\end{array}$ \\
\hline GAT & $y=4.89 \times 10^{6} x+7.96 \times 10^{4}, R^{2}=0.998$ & $0.16-20$ & $0.050 / 0.16$ & $y=24.7 x+39.2, R^{2}=0.998$ & $1.9-60$ & $0.60 / 1.9$ \\
\hline LOM & $y=2.45 \times 10^{6} x+1.11 \times 10^{5}, R^{2}=0.998$ & $0.25-20$ & $0.075 / 0.25$ & $y=26.4 x+10.1, R^{2}=0.999$ & $5.6-60$ & $1.7 / 5.6$ \\
\hline ENO & $y=4.95 \times 10^{6} x-1.12 \times 10^{5}, R^{2}=0.996$ & $0.16-20$ & $0.050 / 0.16$ & $y=14.6 x+6.2, R^{2}=0.999$ & $11-60$ & $3.4 / 11$ \\
\hline FLE & $y=2.77 \times 10^{6} x+3.55 \times 10^{5}, R^{2}=0.999$ & $0.33-20$ & $0.10 / 0.33$ & $y=26.8 x+17.3, R^{2}=0.999$ & $4.3-60$ & $1.3 / 4.3$ \\
\hline ENR & $y=5.16 \times 10^{6} x+2.04 \times 10^{5}, R^{2}=0.999$ & $0.33-20$ & $0.10 / 0.33$ & $y=30.0 x+9.2, R^{2}=0.999$ & $5.0-60$ & $1.5 / 5.0$ \\
\hline PEF & $y=3.22 \times 10^{6} x+1.23 \times 10^{5}, R^{2}=0.999$ & $0.33-20$ & $0.10 / 0.33$ & $y=21.4 x+39.2, R^{2}=0.998$ & $6.6-60$ & $2.0 / 6.6$ \\
\hline
\end{tabular}

and presented in Table 1 . The LODs (3 S/N) and LOQs $(10 \mathrm{~S} / \mathrm{N})$ were calculated and summarized in Table 1, which demonstrated that the LODs obtained in CE-MS were 10 to 40 times lower for different FQs than those in CE-UV. Thus, combining CE-MS of such an improved sensitivity with immunoaffinity

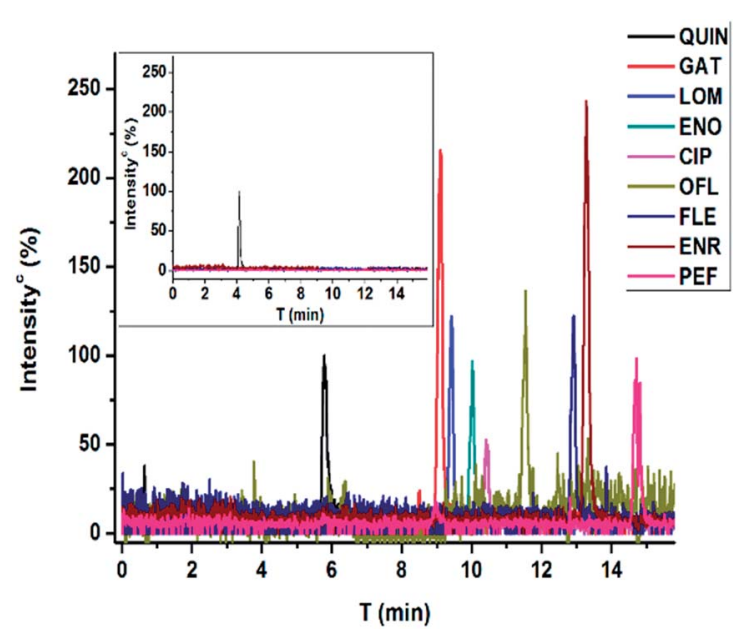

Fig. 4 Electropherograms with MS detection (extracted ion chromatography) for FQs spiked lake water (30 nM for FQs and $5 \mu \mathrm{M}$ for QUIN). The inset is the blank water sample for control. extraction for enrichment provided a potential way to monitor the trace FQs in environmental water.

\subsection{Environmental water analysis}

Organics in environmental samples are generally in trace level and IAE provides an attractive approach to enrich structurally closed analytes in a small volume by off-line being coupled with a chromatographic step for separation. Herein, an evaluated immunoaffinity column was utilized for FQs extraction from spiked lake water and the developed CE-MS was applied for the subsequent qualitative and quantitative analysis. The environmental water samples were simultaneously spiked with eight FQs at different concentrations (30 nM, $15 \mathrm{nM}, 5 \mathrm{nM}$ and $2.5 \mathrm{nM}$, respectively) (Fig. 4). As indicated in Table 2, the presented procedure provided excellent recovery values from $72 \%$ to $112 \%$ and satisfying RSDs between $1.4 \%$ and $17 \%$. Considering the recovery and the difference of the volume of sample $(10 \mathrm{~mL})$ loaded in immunoaffinity column and the volume of redissolved sample $(100 \mu \mathrm{L})$ for CE-MS analysis, the enrichment factor of our columns was about 100 times. The LODs ranging from $1.2 \mathrm{nM}$ for GAT to $5.0 \mathrm{nM}$ for FLE, were comparable or much better than those of reported reverse-phase SPE followed by HPLC-FD, ${ }^{42}$ HPLC-MS, ${ }^{43,44}$ immunoassay $^{10}$ and CE-based method. ${ }^{14,45}$ It's far below the permissible MRL established by European Union. Although the immunoaffinity column was

Table 2 FQs spiked lake water analyzed by off-line coupling of IAE and CE-MS

Spiked environmental water samples $(n=3)$

\begin{tabular}{|c|c|c|c|c|c|c|c|c|c|}
\hline \multirow[b]{2}{*}{ FQs } & \multicolumn{2}{|l|}{$30 \mathrm{nM}$} & \multicolumn{2}{|l|}{$15 \mathrm{nM}$} & \multicolumn{2}{|l|}{$5 \mathrm{nM}$} & \multicolumn{2}{|l|}{$2.5 \mathrm{nM}$} & \multirow[b]{2}{*}{ LOD/LOQ (nM) } \\
\hline & Recovery (\%) & RSD (\%) & Recovery (\%) & $\mathrm{RSD}(\%)$ & Recovery (\%) & $\mathrm{RSD}(\%)$ & Recovery (\%) & RSD (\%) & \\
\hline GAT & 102 & 7.1 & 98.2 & 5.1 & 88.7 & 9.4 & 88 & 13.6 & $1.2 / 4.0$ \\
\hline LOM & 111 & 1.4 & 101 & 7.4 & 108 & 12.1 & 72 & 16.7 & $2.5 / 8.3$ \\
\hline ENO & 99.6 & 2.9 & 103 & 7.9 & 96.7 & 3.2 & $<$ LOD & ND & $3.0 / 10$ \\
\hline CIP & 103 & 2.4 & 108 & 13.2 & 72.0 & 8.3 & $<$ LOD & ND & $3.0 / 10$ \\
\hline OFL & 107 & 12 & 99 & 5.9 & 74 & 15 & $<$ LOD & ND & $3.0 / 10$ \\
\hline FLE & 112 & 9.7 & 75 & 2.1 & $<$ LOD & ND & $<$ LOD & ND & $5.0 / 17$ \\
\hline ENR & 102 & 2.7 & 96.9 & 2.4 & 95.3 & 11.6 & 107 & 9.4 & $1.2 / 4.0$ \\
\hline PEF & 103 & 6.1 & 103 & 5.7 & 88.7 & 4.7 & $<$ LOD & ND & $2.5 / 8.3$ \\
\hline
\end{tabular}


more expensive, it could significantly improve the specificity of extraction step and be used for many times (maybe more than 50 times).

\section{Conclusions}

The presented off-line coupling of IAE with CE-MS was successfully utilized for FQs determination in fresh water collected from a local lake, demonstrated to be stable, sensitive and reliable. A generic polyclonal antibody was prepared and developed to construct an immunoaffinity column for simultaneous extraction and concentration of FQs with similar structure in environmental water. For further qualitative identification and quantitative determination, the hyphenation of CE-MS was applied for high-resolution separation and highsensitivity detection of eight FQs in a single analysis. The satisfactory recoveries of all FQs between $72 \%$ and $112 \%$ shows the usefulness the broad-specificity of the antibody, and the obtained LODs are in range of $1.2 \mathrm{nM}$ to $5.0 \mathrm{nM}$, comparable with that of the widely recommended HPLC-FD. Moreover, the proposed analytical technique can be easily extended to the analysis of FQs in soil or food sample at trace level by adding another sample cleanup step, such as extraction, filtration or centrifugation prior to IAE.

\section{Conflicts of interest}

There are no conflicts to declare.

\section{Acknowledgements}

This work was financially supported by National Natural Science Foundation of China (no. 21575005, 21675004 and 21275009). The author would also like to thank Professor Zhenquan Guo from Lab of Monoclonal Antibody at Peking University for his kind help in antibody preparation.

\section{Notes and references}

1 Z. H. Wang, Y. Zhu, S. Y. Ding, F. Y. He, R. C. Beier, J. C. Li, H. Y. Jiang, C. W. Feng, Y. P. Wan, S. X. Zhang, Z. P. Kai, X. L. Yang and J. Z. Shen, Development of a monoclonal antibody-based broad-specificity ELISA for fluoroquinolone antibiotics in foods and molecular modeling studies of cross-reactive compounds, Anal. Chem., 2007, 79(12), 4471-4483.

2 D. Greenwood, Activity of flumequine against Escherichia coli: in vitro comparison with nalidixic and oxolinic acids, Antimicrob. Agents Chemother., 1978, 13(3), 479-483.

3 D. Cid, S. Piriz, J. A. Ruiz-Santa-Quiteria, J. Valle, S. Garcia, S. Vadillo and R. de la Fuente, In vitro activities of enoxacin, enrofloxacin, sparfloxacin, and ciprofloxacin against Escherichia coli strains isolated from diarrheic lambs and kids, Antimicrob. Agents Chemother., 1994, 38(10), 2469-2470.

4 A. Speltini, M. Sturini, F. Maraschi and A. Profumo, Fluoroquinolone antibiotics in environmental waters: sample preparation and determination, J. Sep. Sci., 2010, 33(8), 1115-1131.

5 C. K. Holtzapple, S. A. Buckley and L. H. Stanker, Immunosorbents coupled on-line with liquid chromatography for the determination of fluoroquinolones in chicken liver, J. Agric. Food Chem., 1999, 47(7), 2963.

6 M. Sturini, A. Speltini, L. Pretali, E. Fasani and A. Profumo, Solid-phase extraction and HPLC determination of fluoroquinolones in surface waters, J. Sep. Sci., 2009, 32(17), 3020-3028.

7 K. He and L. Blaney, Systematic optimization of an SPE with HPLC-FLD method for fluoroquinolone detection in wastewater, J. Hazard. Mater., 2015, 282(4), 96-105.

8 D. Ašperger, V. Tišler, M. Zrnčić, D. M. Pavlović, S. Babić, A. J. M. Horvat and M. Kaštelan-Macan, HPLC-DAD-FLD determination of veterinary pharmaceuticals in pharmaceutical industry wastewater with precolumn derivatization using fluorescamine, Chromatographia, 2014, 77(15-16), 1059-1066.

9 S. M. Khor, G. Liu, J. R. Peterson, S. G. Iyengar and J. J. Gooding, An electrochemical immunobiosensor for direct detection of veterinary drug residues in undiluted complex matrices, Electroanalysis, 2011, 23(8), 1797-1804.

10 Z. Zhang, J.-f. Liu, T.-t. Feng, Y. Yao, L.-h. Gao and G.-b. Jiang, Time-Resolved Fluoroimmunoassay as an Advantageous Analytical Method for Assessing the Total Concentration and Environmental Risk of Fluoroquinolones in Surface Waters, Environ. Sci. Technol., 2013, 47(1), 454-462.

11 B. Chen, W. Wang and Y. Huang, Cigarette filters as adsorbents of solid-phase extraction for determination of fluoroquinolone antibiotics in environmental water samples coupled with high-performance liquid chromatography, Talanta, 2012, 88(1), 237-243.

12 M. Lombardoagüí, C. Crucesblanco, A. M. Garcíacampaña and L. Gámizgracia, Multiresidue analysis of quinolones in water by ultra-high performance liquid chromatography with tandem mass spectrometry using a simple and effective sample treatment, J. Sep. Sci., 2014, 37(16), 2145.

13 E. Gracia-Lor, J. V. Sancho and F. Hernández, Multi-class determination of around 50 pharmaceuticals, including 26 antibiotics, in environmental and wastewater samples by ultra-high performance liquid chromatography-tandem mass spectrometry, J. Chromatogr. A, 2011, 1218(16), 2264.

14 A. V. Herrera-Herrera, J. Hernández-Borges, T. M. BorgesMiquel and M. Á. Rodríguez-Delgado, Dispersive liquidliquid microextraction combined with nonaqueous capillary electrophoresis for the determination of fluoroquinolone antibiotics in waters, Electrophoresis, 2010, 31(20), 3457-3465.

15 A. V. Herrera-Herrera, L. M. Ravelo-Perez, J. HernandezBorges, M. M. Afonso, J. A. Palenzuela and M. A. RodriguezDelgado, Oxidized multi-walled carbon nanotubes for the dispersive solid-phase extraction of quinolone antibiotics from water samples using capillary electrophoresis and large volume sample stacking with polarity switching, J. Chromatogr. A, 2011, 1218(31), 5352-5361. 
16 M. Ferdig, A. Kaleta, T. D. Vo and W. Buchberger, Improved capillary electrophoretic separation of nine (fluoro) quinolones with fluorescence detection for biological and environmental samples, J. Chromatogr. A, 2004, $1047(2), 305$.

17 M. D. Luaces, J. L. Urraca, M. C. Pérez-Conde, N. C. M. Alfonso, A. C. Valdés-González, A. M. Gutiérrez and M. C. Moreno-Bondi, Chemiluminescence analysis of enrofloxacin in surface water using the tris(1,10phenanthroline)-ruthenium(II)/peroxydisulphate system and extraction with molecularly imprinted polymers, Microchem. J., 2013, 110(9), 458-464.

18 Y. Deng, N. Gasilova, L. Qiao, Y. L. Zhou, X. X. Zhang and H. H. Girault, Highly sensitive detection of five typical fluoroquinolones in low-fat milk by field-enhanced sample injection-based CE in bubble cell capillary, Electrophoresis, 2014, 35(23), 3355.

19 M. Lillenberg, S. Yurchenko, K. Kipper, K. Herodes, V. Pihl, K. Sepp, R. Lõhmus and L. Nei, Simultaneous determination of fluoroquinolones, sulfonamides and tetracyclines in sewage sludge by pressurized liquid extraction and liquid chromatography electrospray ionization-mass spectrometry, J. Chromatogr. A, 2009, 1216(32), 5949.

20 J. Radjenović, A. Jelić, M. Petrović and D. Barceló, Determination of pharmaceuticals in sewage sludge by pressurized liquid extraction (PLE) coupled to liquid chromatography-tandem mass spectrometry (LC-MS/MS), Anal. Bioanal. Chem., 2009, 393(6-7), 1685-1695.

21 W. Bąk, I. M. Choma and B. Majer-Dziedzic, Matrix solidphase dispersion combined with thin-layer chromatography-direct bioautography for determination of flumequine residues in milk: improvement of the method, J. Liq. Chromatogr. Relat. Technol., 2011, 34(10-11), 920-927.

22 D. Li, Q. Yang, Z. Wang, R. Su, X. Xu and H. Zhang, Determination of fluoroquinolones in blood by matrix solid-phase dispersion extraction and CE, J. Sep. Sci., 2011, 34(7), 822-829.

23 N. Dorivalgarcía, A. Zafragómez, F. J. Caminosánchez, A. Navalón and J. L. Vílchez, Analysis of quinolone antibiotic derivatives in sewage sludge samples by liquid chromatography-tandem mass spectrometry: comparison of the efficiency of three extraction techniques, Talanta, 2013, 106(6), 104-118.

24 A. Speltini, M. Sturini, F. Maraschi, A. Profumo and A. Albini, Microwave-assisted extraction and determination of enrofloxacin and danofloxacin photo-transformation products in soil, Anal. Bioanal. Chem., 2012, 404(5), 1565-1569.

25 E. Cohen, R. J. Maxwell and D. J. Donoghue, Automated multi-residue isolation of fluoroquinolone antimicrobials from fortified and incurred chicken liver using on-line microdialysis and high-performance liquid chromatography with programmable fluorescence detection, J. Chromatogr. B: Biomed. Sci. Appl., 1999, 724(1), 137.

26 M. C. Hennion and V. Pichon, Immuno-based sample preparation for trace analysis, J. Chromatogr. A, 2003, 1000(1-2), 29-52.

27 L. E. Vera-Avila, B. P. Márquez-Lira, M. Villanueva, R. Covarrubias, G. Zelada and V. Thibert, Determination of carbofuran in surface water and biological tissue by sol-gel immunoaffinity extraction and on-line preconcentration/ HPLC/UV analysis, Talanta, 2012, 88(1), 553.

28 Z. L. Xu, H. Deng, H. T. Lei, Y. M. Jiang, K. Campbell, Y. D. Shen, J. Y. Yang, H. Wang and Y. M. Sun, Development of a broad-specificity monoclonal antibody-based immunoaffinity chromatography cleanup for organophosphorus pesticide determination in environmental samples, J. Agric. Food Chem., 2012, 60(23), 5847-5852.

29 H. X. Chen, Q. P. Deng, L. W. Zhang and X. X. Zhang, Quantification of testosterone and epitestosterone in biological samples by capillary electrophoresis with immunoaffinity extraction, Talanta, 2009, 78(2), 464-470.

30 N. Delaunay-Bertoncini and M. C. Hennion, Immunoaffinity solid-phase extraction for pharmaceutical and biomedical trace-analysis-coupling with HPLC and CE-perspectives, Int. J. Pharm. Res. Biomed. Anal., 2004, 34(4), 717-736.

31 S. Soltani and A. Jouyban, Biological sample preparation: attempts on productivity increasing in bioanalysis, Bioanalysis, 2014, 6(12), 1691-1710.

32 K. Pavšič-Vrtač, S. Ojanperä, J. Apajalahti, K. Šrimpf and G. Tavčar-Kalcher, Analytical procedures for the determination of aflatoxin b1 in eggs of laying hens using immunoaffinity columns and liquid chromatography with post-column derivatisation and fluorescence detection, Food Anal. Method, 2014, 7(9), 1917-1924.

33 H. Z. Senyuva and J. Gilbert, Immunoaffinity column cleanup techniques in food analysis: a review, J. Chromatogr. B: Anal. Technol. Biomed. Life Sci., 2010, 878(2), 115-132.

34 C. K. Holtzapple, S. A. Buckley and L. H. Stanker, Determination of four fluoroquinolones in milk by on-line immunoaffinity capture coupled with reversed-phase liquid chromatography, J. AOAC Int., 1999, 82(3), 607.

35 T. L. Goodfriend, L. Levine and G. D. Fasman, Antibodies To Bradykinin And Angiotensin: A Use Of Carbodiimides In Immunology, Science, 1964, 144(3624), 1344.

$36 \mathrm{H}$. X. Chen and X. X. Zhang, Antibody development to testosterone and its application in capillary electrophoresisbased immunoassay, Electrophoresis, 2008, 29(16), 3406-3413.

37 M. P. Zhao, Y. Z. Li, Z. Q. Guo, X. X. Zhang and W. B. Chang, A new competitive enzyme-linked immunosorbent assay (ELISA) for determination of estrogenic bisphenols, Talanta, 2002, 57(6), 1205-1210.

38 J. D. Beatty, B. G. Beatty and W. G. Vlahos, Measurement of monoclonal antibody affinity by non-competitive enzyme immunoassay, J. Immunol. Methods, 1987, 100(1-2), 173-179.

39 J. J. Pratt, Steroid immunoassay in clinical-chemistry, Clin. Chem., 1978, 24(11), 1869-1890.

40 M. Y. Piñero, R. Garrido-Delgado, R. Bauza, L. Arce and M. Valcárcel, Easy sample treatment for the determination of enrofloxacin and ciprofloxacin residues in raw bovine milk by capillary electrophoresis, Electrophoresis, 2012, 33(19-20), 2978-2986.

41 A. Juan-García, G. Font and Y. Picó, Determination of quinolone residues in chicken and fish by capillary electrophoresis-mass spectrometry, Electrophoresis, 2006, 27(11), 2240-2249. 
42 M. Petrovic, M. Gros and D. Barcelo, Multi-residue analysis of pharmaceuticals in wastewater by ultra-performance liquid chromatography-quadrupole-time-of-flight mass spectrometry, J. Chromatogr. A, 2006, 1124(1-2), 68-81.

43 M. P. Hermo, E. Nemutlu, S. Kır, D. Barrón and J. Barbosa, Improved determination of quinolones in milk at their MRL levels using LC-UV, LC-FD, LC-MS and LC-MS/MS and validation in line with regulation 2002/657/EC, Anal. Chim. Acta, 2008, 613(1), 98-107.
44 O. Szerkus, J. Jacyna, A. Gibas, M. Sieczkowski, D. Siluk, M. Matuszewski, R. Kaliszan and M. J. Markuszewski, Robust HPLC-MS/MS method for levofloxacin and ciprofloxacin determination in human prostate tissue, J. Pharm. Biomed. Anal., 2017, 132, 173-183.

45 M. Ferdig, A. Kaleta, T. D. T. Vo and W. Buchberger, Improved capillary electrophoretic separation of nine (fluoro)quinolones with fluorescence detection for biological and environmental samples, J. Chromatogr. A, 2004, 1047(2), 305-311. 